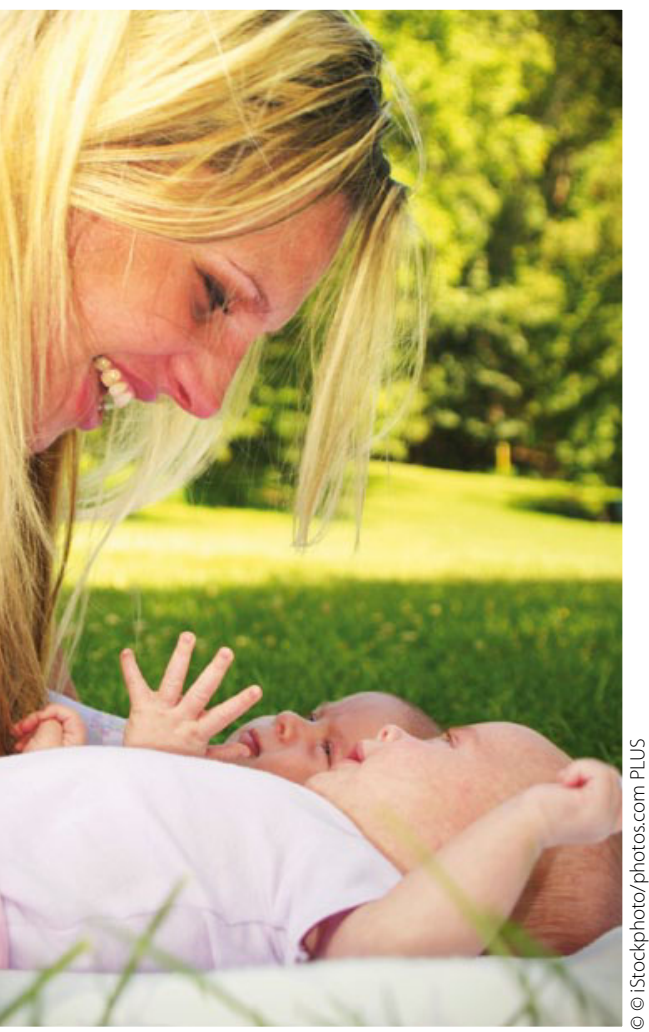

Zwillingsgeburten sind heute oft Folge einer Hormonbehandlung.

\title{
Zwillingsmütter sind robuster
}

Was bedeutet die Geburt von Zwillingen für die Mütter? Hier sind zwei Antworten denkbar: Die Zwillingsgeburt "geht an die Substanz", die Frauen sind danach krankheitsanfälliger und sterben $u$. $U$. auch früher als Frauen, die nur Einzelkinder zu Welt gebracht haben. Oder aber: Es sind besonders gesunde und robuste Frauen, die zu Zwillingsschwangerschaften neigen.

_ Offensichtlich trifft die zweite Möglichkeit zu. Dies ergab jedenfalls eine Studie von Wissenschaftlern an der Universität Utah in Salt Lake City (USA), die den Zusammenhang zwischen Zwillingsgeburten und der Gesundheit und Fruchtbarkeit der Mütter untersuchten.

Die Daten von 59000 Frauen, die im 19. Jahrhundert, zwischen 1807 und 1899 in Nordamerika lebten und älter als 50 Jahre wurden, wurden mithilfe von proportionalen Risiko- und Regressionsanalysen ausgewertet.

4603 dieser Frauen hatten Zwillinge geboren. Der Datenvergleich zeigte, dass die Zwillingsmütter im Durchschnitt länger lebten als die anderen Frauen, die immer nur Einlinge zur Welt gebracht hatten. Darüber hinaus bekamen die Zwillingsmütter mehr Kinder und waren länger fruchtbar. Das Zeitintervall zwischen den Geburten war kürzer.

\section{Kommentar}

Nach Meinung der Autoren könne man daraus nicht schließen, dass Zwillingsgeburten zu einem längeren Leben führten. Eher richtig sei, dass gesündere Frauen mehr Zwillinge gebären. Eine Übertragung der Ergebnisse auf die Gegenwart ist allerdings schwierig. Viele Zwillingsgeburten gehen heute auf eine Hormonbehandlung zurück. Dadurch hat sich in den letzten 30 Jahren in Deutschland die Zahl der Zwillingsgeburten verdoppelt.

K.MALBERG =

- S. L. Robson, K. R. Smith

(Department of Anthropology, University of Utah, Salt Lake City, UT, USA; e-mail: robson@ umnh.utah.edu) Twinning in humans: maternal heterogeneity in reproduction and survival. Published before print on 11 Mai 2011 in Proc. R. Soc. B. doi: rspb.2011.0573v1-rspb20110573

\section{Kann regelmäßige Wirbelsäulenmanipulation Nackenschmerzen verhindern?}

\begin{abstract}
Wirbelsäulenmanipulationen sollen bei Nackenschmerzen nicht nur im Akutzustand helfen, sondern auch nach dem Abklingen der Beschwerden Rezidive verhindern. Die Wirksamkeit dieser Rezidivprophylaxe wurde bisher kaum wissenschaftlich untersucht.
\end{abstract}

- Kanadische Chiropraktiker randomisierten 98 Patienten mit Nackenschmerzen in drei Gruppen. Nach Abklingen der akuten Beschwerden wurden sie zehn Monate lang entweder regelmäßig mit Wirbelsäulenmanipulationen oder mit ebensolchen Manipula- tionen plus einem physiotherapeutischen Übungsprogramm, oder gar nicht behandelt. Am Ende dieser präventiven Maßnahmen zeigten sich für keinen der Studienendpunkte wie z. B. Schmerz, Funktion oder Behinderungsgrad signifikante Gruppendifferenzen. Die Autoren schließen daraus, dass die präventive Wirbelsäulenmanipulation zur Verhütung von Nackenschmerzrezidiven nicht wirkungsvoll ist.

\section{Kommentar}

Weltweit verdienen Chiropraktiker viel Geld mit dieser sogenannten Maintenance Therapy. Daher ist es kaum zu glauben, dass bislang außer einer kleinen Pilotstudie keine Untersuchungen zu diesem Thema vorlagen. Die hier vorgestellte Studie ist gut gemacht und zeigt die Unwirksamkeit dieses Vorgehens recht eindeutig. Ob jetzt allerdings die Chiropraktiker aller Länder auf diese Einkommensquelle verzichten werden, wage ich zu bezweifeln.

E. ERNST . 